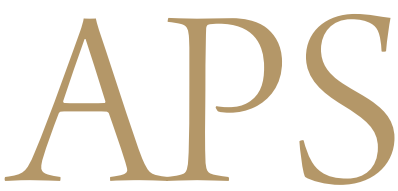

Archives of Plastic Surgery

\title{
Comparison Study of the Use of Absorbable and Nonabsorbable Materials as Internal Splints after Closed Reduction for Nasal Bone Fracture
}

\author{
Chang Ryul Yi, Young Joon Kim, Hoon Kim, Sang Hyun Nam, Young Woong Choi \\ Department of Plastic and Reconstructive Surgery, Inje University Sanggye Paik Hospital, Inje University College of Medicine, Seoul, Korea
}

Background The authors sought to compare the use of the nonabsorbable polyvinyl alcohol sponge (PVA, Merocel) and absorbable synthetic polyurethane foam (SPF, Nasopore Forte plus) as intranasal splints after closed reduction of fractured nasal bones during the hospitalization period.

Methods The medical records of 111 patients who underwent closed reduction for nasal bone fracture at Sanggye Paik Hospital, Inje University College of Medicine, from 2012 to 2013 were reviewed retrospectively. PVA (group A) or SPF (group B) was packed as an internal splint after closed reduction. The efficacy of the materials was compared and statistically analyzed.

Results PVA was used in 82 patients, and SPF was used in 29 patients. The patients in group B complained significantly more of nasal pain on the first day after operation than the patients in group A. Headaches on the operation day were significantly more painful in group B than in group $A$. Bleeding on the fourth postoperative day was significantly reduced in group $B$ as compared to group $A$. The patients in group B exhibited significantly more intensive nasal obstruction on the operation day and the following day than the patients in group A. However, on the third and fourth postoperative days, the nasal obstruction in group B was less than that in group $A$. The pain and bleeding related to the packing material was significantly reduced in group B as compared to group A.

Conclusions The use of SPF as an absorbable packing material is a reasonable substitute for the traditional nonabsorbable material.

\section{Keywords Nasal bone / Splints / Facial bones}

Correspondence: Young Joon Kim Department of Plastic and Reconstructive Surgery, Inje University Sanggye Paik Hospital, Inje University College of Medicine, 1342 Dongil-ro, Nowon-gu, Seoul 139-707, Korea Tel: +82-2-950-1048 Fax: +82-2-932-6373 E-mail:S2639@paik.ac.kr

\section{INTRODUCTION}

Nasal bone fracture is the most common fracture in facial trau$\mathrm{ma}[1,2]$ and the third most commonly fractured human bone [2]. The general treatment for fracture is closed reduction, which requires an internal splint in the nostril to stabilize the reduced bone fragments [1]. Because the functions of the internal splint include the stabilization of the bone segments, epistaxis control, prevention of synechia formation, and discharge absorption [1], the splint material is important. Furthermore, nasal packing in the internal splint can cause painful side effects, including dyspnea, sore throat, and headache, and many studies concerning the packing materials have been reviewed in the literature [1,3-9]. 
Typically, nasal packing materials include Vaseline gauze and polyvinyl alcohol sponge (Merocel, Medtronic Xomed Surgical Products, Jacksonville, FL, USA) [1,7]. These materials are nonabsorbable; thus, the nasal airway is completely obstructed throughout the packing time, potentially leading to complications. To compensate for the shortcomings of these packing materials, absorbable materials, including synthetic polyurethane foam (Nasopore Forte plus, Polyganics Rosenburglaan, Groningen, The Netherlands), are increasingly being used $[1,7]$.

One study compared nonabsorbable material with biodegradable material only in endoscopic sinus surgery [7], but the present study was the first trial using nonabsorbable material during nasal bone fracture surgery.

In this study, the authors sought to compare the use of the nonabsorbable polyvinyl alcohol sponge (PVA) and absorbable synthetic polyurethane foam (SPF) in intranasal splints after closed reduction of fractured nasal bones during the hospitalization period.

\section{METHODS}

After obtaining approval from the institutional review board, the medical records of 111 patients who underwent closed reduction of a nasal bone fracture were reviewed retrospectively. Only pure nasal bone fracture patients who did not exhibit any other facial bone fractures were included. All of the patients were admitted and underwent surgery between May 2012 and April 2013. PVA was packed into one group of patients (group A) as an internal splint, and SPF was packed into the other group (group B) as an internal splint after closed reduction. The efficacy of the materials was compared and statistically analyzed.

The operation was performed under general anesthesia. Before nasal reduction, epinephrine-soaked gauzes were packed into both nostrils for 10 minutes to control epistaxis. After the fractured nasal bone and septum were reduced in situ, an internal splint of PVA or SPF was applied. Eight cm of PVA or SPF spreading ointment (Terramycin Ophthalmic Ointment, Pfizer Inc., New York, NY, USA) was packed in the nostrils without manipulation. The intranasal packing was removed on the fourth day after surgery. The external nasal splint (External Nasal Splint Singles, Medtronic Xomed Surgical Products, Jacksonville, FL, USA) was maintained for 1 month after surgery.

The authors compared the efficacy and shortcomings of each packing material. Nasal pain and headache were assessed every day until the packing was removed using the visual analog scale (VAS) from 0 to 10. Every day, the amount of bleeding and the severity of nasal obstruction were estimated according to $4 \mathrm{lev}$ els: very much, much, little, and none. Additionally, the pres- ence of sleep disturbances, postnasal drip, and other symptoms was assessed at removal.

The medical records from postoperative follow-up visits were also reviewed. The patients visited an outpatient department 2 weeks after discharge. The authors analyzed patients' complaints about postoperative complications and satisfaction. Nasal obstruction and discharge was classified into 4 levels: very severe, severe, mild, and none. The satisfaction of the patient was divided into 4 levels with regard to aesthetics and olfactory sense: very unsatisfactory, unsatisfactory, satisfactory, and very satisfactory. The demand for re-operation was also classified into 4 levels: very high demand, high demand, low demand, and no demand.

Complications in hospital days, including sleep disturbance, postnasal drip, pain at removal, and bleeding at removal, were statistically analyzed with a chi-squared test. The independent $t$ test was used for the statistical analysis of all other data. Further, $\mathrm{P}<0.05$ was considered statistically significant. All statistical analyses were performed with the SAS software (ver. 5.1, SAS Institute, Cary, NC, USA).

\section{RESULTS}

One hundred eleven patients with nasal bone fracture were reviewed. PVA was packed into 82 of the patients (group A), and SPF was used in 29 patients (group B). Group A included 66 males and 16 females with a mean age of $30.0 \pm 14.56$ years. Group B consisted of 20 males and 9 females with a mean age of $25.7 \pm 14.38$ years. There is no significant difference in sex and age between the two groups $(\mathrm{P}=0.20, \mathrm{P}=0.69)$ (Table 1).

Nasal pain from the operation day to the fourth postoperative day (days $0,1,2,3$, and 4), based on VAS scores, was 4.9, 3.7, 2.3, 1.4, and 0.8 in group A and 6.1, 5.0, 3.1, 1.3, and 0.4 in group B, respectively. There was no significant difference between the two groups, except on the first postoperative day $(\mathrm{P}=0.032)$. The patients in group $\mathrm{B}$ complained of more severe nasal pain on the first day after operation (Fig. 1).

The VAS scores for headaches were 5.8, 4.9, 3.2, 1.7, and 1.1 in

\begin{tabular}{|c|c|c|c|}
\hline & PVA $(n=82)$ & SPF $(n=29)$ & P-value \\
\hline Sex & & & $0.20^{\mathrm{a})}$ \\
\hline Male & 66 (80.5) & $20(69.0)$ & \\
\hline Female & 16 (19.5) & $9(31.0)$ & \\
\hline Age (mean) & 30 & 25.7 & $0.69^{b)}$ \\
\hline \multicolumn{4}{|c|}{$\begin{array}{l}\text { Values are presented as number (\%). } \\
\text { PVA, polyvinyl alcohol sponge; SPF, synthetic polyurethane foam. } \\
{ }^{a} \text { Chi-square test; }{ }^{\prime \prime l} \text { Independent t-test. }\end{array}$} \\
\hline
\end{tabular}




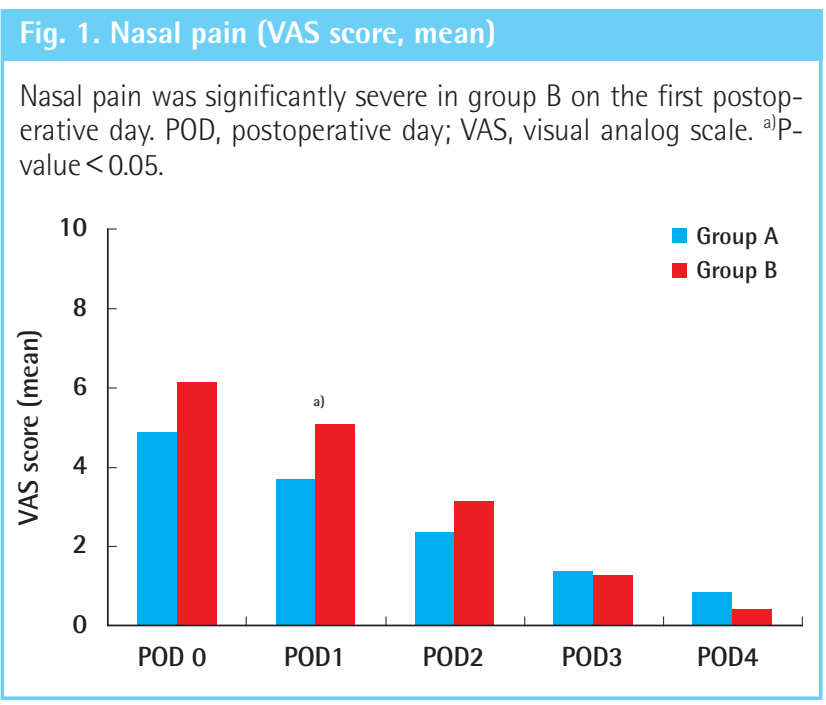

Fig. 2. Headache (VAS score, mean)

Headache was significantly more severe in group B on the operation day. POD, postoperative day; VAS, visual analog scale. ${ }^{\text {a) }}$-value < 0.05 .

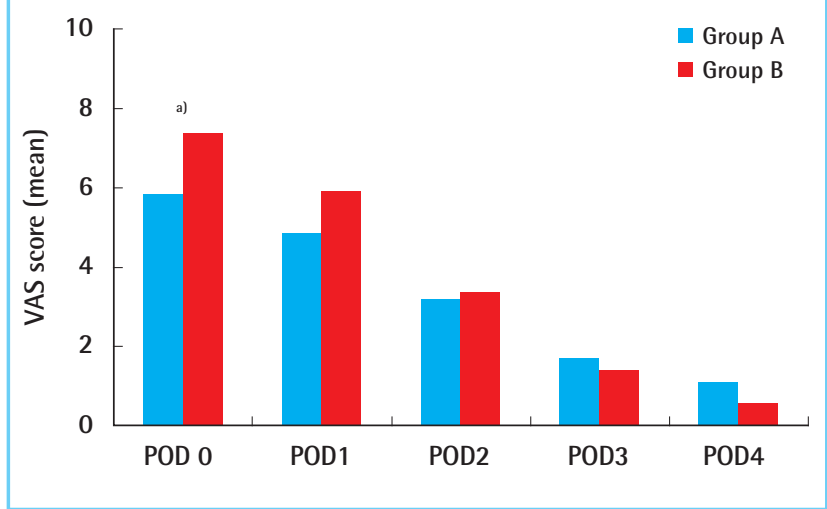

group A and 7.4, 5.9, 3.4, 1.4, and 0.6 in group B from the operation day to the fourth postoperative day. Only the headaches on the operation day were significantly more painful in the group $\mathrm{B}$ patients as compared to the group A patients $(\mathrm{P}=0.031)$ (Fig. 2).

The amount of bleeding on each postoperative day (days 0,1 , 2,3 , and 4), which was classified into 4 levels, was 1.9, 1.5, 1.0, 0.6 , and 0.4 in group $A$ and $1.5,1.2,0.9,0.5$, and 0.2 in group $B$, respectively. Bleeding on the fourth postoperative day was significantly reduced in group $\mathrm{B}$ as compared to group $\mathrm{A}(\mathrm{P}=0.013)$ (Fig. 3).

The intensity of nasal obstruction, which was classified into 4 levels, was 2.7, 2.7, 2.6, 2.3, and 2.0 in group A and 3.5, 3.3, 2.6, 1.5 , and 1.0 in group $B$ on postoperative days $0,1,2,3$, and 4 , respectively. The use of SPF resulted in significantly more obstructions on the operation day and the following day $(\mathrm{P}=0.031, \mathrm{P}<$ $0.001)$. On the third and fourth postoperative days, obstruction was significantly reduced in the PVA group $(\mathrm{P}<0.001, \mathrm{P}<$

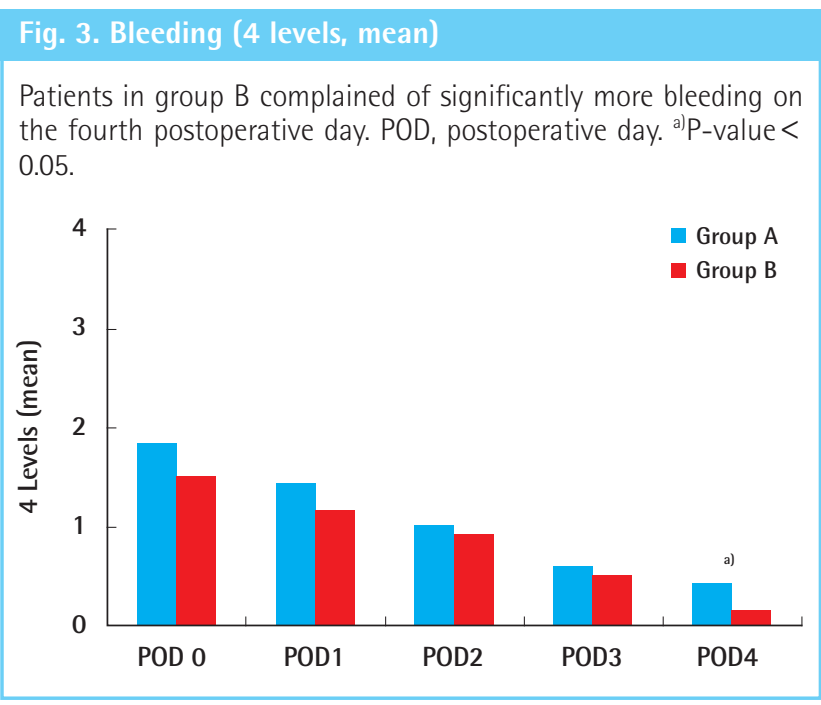

Fig. 4. Obstruction (4 levels, mean)

Group B felt significantly more severe nasal obstruction on the operation day and the first postoperative day; otherwise, group A complained of more severe obstruction on the third and fourth postoperative days. POD, postoperative day. ${ }^{\text {a) }} \mathrm{P}$-value $<0.05$.

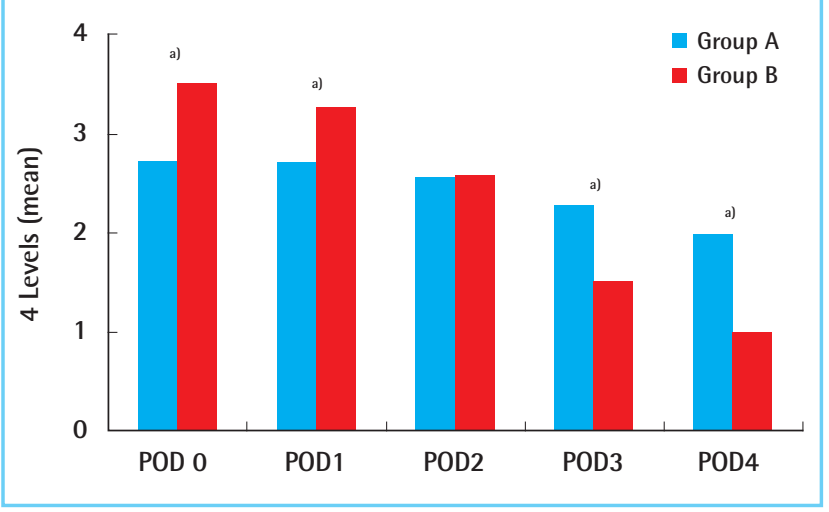

0.001) (Fig. 4).

Fifty nine patients (72\%) in group A and 22 patients (76\%) in group B complained of sleep disturbance, but this difference was not statistically significant. Thirty six patients (44\%) in group A and 16 patients (55\%) in group B complained of postnasal drip, which was not statistically significant. Pain and bleeding related to the removal of the packing material were reported by 72 patients ( $88 \%)$ and 53 patients $(65 \%)$ in group A, respectively, and by 10 patients (34\%) and 3 patients (10\%) in group $\mathrm{B}$, respectively, and these differences were statistically significant $(\mathrm{P}<0.001, \mathrm{P}<0.001)$ (Table 2).

After discharge, 57 of 111 patients were followed. Group A exhibited less severe nasal obstruction and discharge. However, group A also exhibited less satisfaction with the surgery and a greater demand for re-operation. Nonetheless, the differences between the groups were not statistically significant (Fig. 5). 
Table 2. Rates of other complications during the hospitalization period and symptoms at removal

\begin{tabular}{|lccc|}
\hline & Group A & Group B & P-value $^{\text {a) }}$ \\
\hline Sleep disturbance & $59(72)$ & $22(76)$ & 0.690 \\
Postnasal drip & $36(44)$ & $16(55)$ & 0.300 \\
Pain at removal & $72(88)$ & $10(34)$ & $<0.001$ \\
Bleeding at removal & $53(65)$ & $3(10)$ & $<0.001$ \\
\hline $\begin{array}{l}\text { Values are presented as number (\%). } \\
\text { a)Chi-square test. }\end{array}$ & & \\
\hline
\end{tabular}

\section{DISCUSSION}

After closed reduction of nasal bone fracture, an internal splint is necessary to support the reduced bone fragments. To date, nasal packing has been the only method for internal splinting. Because of the other roles of the nasal packing material, such as hemostasis, discharge absorption, and synechiae prevention, intranasal packing is considered an essential procedure after closed reduction [1]. Thus, nasal packing is applied despite the nasal airway obstruction that can result in painful complications such as headache, dry mouth, or cough. The pain and epistaxis that can follow the removal of packing material are the main complications of the procedure. Significant efforts have been made to decrease these problems $[5,8]$, but no satisfactory method has been identified to date.

One potential solution is the use of biodegradable products. One of these absorbable materials is SPF, which is composed of 1, 4-butanediisocybnate and co-polyether ester urethane. A polyurethane bond has initial compressive mechanical properties, and the hydrophilic component leads to water uptake and fragmentation [1]. If it takes water, SPF slowly degrades to water and carbon dioxide, which are harmless. Because SPF can contain a great quantity of water, it absorbs blood and exudate to reduce discharge as packing material. The property by which SPF maintains its shape for 48 hours and consumes water keeps the wound wet and leads to a hemostatic effect upon wound compression. SPF degrades and is eliminated spontaneously; therefore, rebleeding and damage of the mucosa is minimal when the material is removed [4].

Thus far, there is some controversy over the use of this absorbable material for intranasal packing. One of the controversies is the concern about the ability of supporting the reduced nasal bone fragments. Because SPF maintains its shape only for 48 hours, a fractured bone fragment can be dislocated and the surgical outcome may be worse. However, Woo et al. [10] reported that the duration of nasal packing did not correlate with the outcome in the analysis of 413 cases. The short period of maintenance of the shape does not seem to be a problem in the use of

\section{Fig. 5. Follow-up}

All the symptoms and satisfactions do not show any significant difference between the two groups.

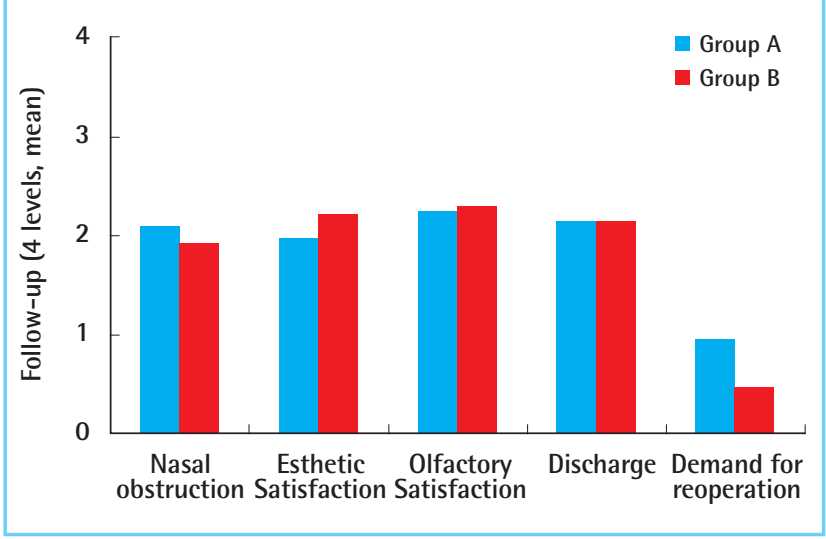

SPF as an internal splint, but further study about the surgical outcome is needed.

Another controversy is the efficacy in hemostasis. Wang et al. [7] reported that SPF provides less compression pressure than traditional packing materials and was, therefore, less useful for hemostasis in endoscopic sinus surgery. Moon et al. [1] reported that SPF exhibited a hemostatic effect similar to that of other materials used in nasal bone fracture surgery. Huang et al. [11] observed less fibrosis and ototoxicity in the middle ear in the SPF group than in the group using the traditional material, absorbable gelatin sponge.

The use of SPF in endoscopic sinus surgery or otosurgery differs from its use in nasal bone surgery due to the mechanism of mucosal injury. However, few studies have reported its use in nasal bone fracture surgery. Closed reduction of nasal bone fractures usually causes only superficial injuries to the mucosa. The amount of bleeding is relatively limited compared with endoscopic sinus surgery, which requires the incision of the mucosa [1]. Several studies report the inferiority of SPF in other surgeries, but Moon et al. [1] observed a hemostatic ability of SPF that was similar to the traditional packing materials used in nasal bone fracture surgery.

The authors demonstrated that SPF can provide equivalent or superior hemostatic effects in nasal bone surgery as compared to PVA. The amount of bleeding was similar between the PVA group and the SPF group from the operation day to the third postoperative day. On the fourth postoperative day, the SPF group showed a significantly smaller volume of bleeding, which implies its superiority with respect to the hemostatic effect. From the perspective of hemostasis after closed reduction, SPF can replace PVA as an internal splint.

Considering the other roles of an internal splint, absorption 
ability is an important property when the surgeon chooses packing material. In this study, the ability to absorb discharge was inferred through the rate of complaints about the postnasal drip. That the rate of patients who complained of postnasal drip had no statistically significant difference proved that the SPF has similar absorption properties to PVA. Further, it can be concluded that SPF absorbs as much discharge as PVA after nasal bone surgery.

Nasal pain and headache are the main complications of intranasal packing. The outward compression can cause nasal pain. The obstruction of the opening of the frontal sinus may result in headache. Therefore, the absorption of the packing material is expected to reduce the mechanical compression and retain the passage of the frontal sinus. Unexpectedly, in our study, nasal pain and headache during the packing period were not relieved in the SPF group as compared to the PVA group, although the volume of the material was reduced by absorption. The more painful symptoms reported on the operation day and the following day in the SPF group, as compared to the PVA group, can be explained by the significantly more intensive obstruction caused by SPF on those days.

Symptoms at the removal of the material were reported less frequently in the SPF group. Bleeding and pain related to the removal of the packing are some of the most disturbing symptoms for patients [1]. Despite the initial compressive properties on the operation day and the first postoperative day, SPF begins to dissolve on the second day $[1,2]$. Our study also demonstrated the significantly decreased obstruction in the SPF group as compared to the PVA group after the third postoperative day. This characteristic of SPF significantly relieves the painful symptoms associated with the removal of the packing material.

Due to the small sample size, the two materials exhibited no significant difference in the patient's satisfaction with the surgery or postoperative complications.

The small cohort of patients who received SPF is a major limitation of our study. Long-term follow-up studies in a larger patient group are necessary. The authors plan the prospective study including the outcome of the surgery and long-term follow-up of the sequelae.

In conclusion, the absorbable packing material SPF is believed to represent a reasonable substitute for traditional nonabsorbable materials. SPF shows a similar hemostatic effect and absorption of discharge as a packing material as compared to the traditional material, PVA. Complications in hospital days were not more severe than in the case of PVA. In particular, with respect to the complications related to packing removal, SPF was proven to be an appropriate packing material for nasal bone fracture.

\section{REFERENCES}

1. Moon SH, Baek SO, Jung SN, et al. Efficacy of biodegradable synthetic polyurethane foam for packing nasal bone fractures. J Craniofac Surg 2012;23:1848-50.

2. Mondin V, Rinaldo A, Ferlito A. Management of nasal bone fractures. Am J Otolaryngol 2005;26:181-5.

3. Jin $\mathrm{KH}$, Choi JS, Kim YH, et al. Use of triamcinolone-impregnated nasal packing following endoscopic sinus surgery. J Rhinol 2012;19:119-22.

4. Ahn SM, Kim SS. Clinical application of polyether ester urethane in endonasal dacryocystorhinostomy. J Korean Ophthalmol Soc 2012;53:743-8.

5. Rhee SC, Kim JS. A simple method of fabricating nasal packing armed with ventilation tube. J Craniofac Surg 2008; 19:1385-6.

6. Lee SH, Shim WS, Shin SO, et al. Endoscopic endonasal reduction of medial blow out fracture using Nasopore Forte(R). Korean J Otorhinolaryngol-Head Neck Surg 2011;54:61722.

7. Wang YP, Wang MC, Chen YC, et al. The effects of Vaseline gauze strip, Merocel, and Nasopore on the formation of synechiae and excessive granulation tissue in the middle meatus and the incidence of major postoperative bleeding after endoscopic sinus surgery. J Chin Med Assoc 2011;74:16-21.

8. Kim HY, Kim SR, Park JH, et al. The usefulness of nasal packing with vaseline gauze and airway silicone splint after closed reduction of nasal bone fracture. Arch Plast Surg 2012; 39:612-7.

9. Cote DW, Wright ED. Triamcinolone-impregnated nasal dressing following endoscopic sinus surgery: a randomized, double-blind, placebo-controlled study. Laryngoscope 2010; 120:1269-73.

10. Woo KS, Lee SH, Shin WH, Cho PD. Outcome analysis of nasal bone fracture with regard to the duration of nasal packing. In: Abstract book, 70th Congress of the Korean Society of Plastic and Reconstructive Surgeons; 2012 Nov 9-11; Seoul, Korea. p. 60.

11. Huang G, Chen X, Jiang H. Effects of NasoPore packing in the middle ear cavity of the guinea pig. Otolaryngol Head Neck Surg 2011;145:131-6. 